\title{
Generalized Rheological Model of the Unconsolidated Marine Sediments with Internal Friction and Effective Compressibility
}

\author{
V. A. Lisyutin \\ Sevastopol State University, Sevastopol, Russian Federation \\ vlisiutin@mail.ru
}

Introduction. The quasi-two-phase theory of propagation of an elastic wave in the unconsolidated marine sediments is represented. The longitudinal and shear waves can propagate in marine sediments. The phase velocities, the attenuation coefficients and their frequency dependences are the acoustic properties of these waves. It is shown experimentally that the in dry granular media, the attenuation coefficient is directly proportional to frequency. Whereas in the saturated media, the deviations from this law are noted whence it follows that there are two physical loss mechanisms: the internal and viscous friction.

Data and methods. The medium in a dry state is represented as a generalized Kelvin-Voigt element consisting of a spring and a springpot that is an element combining the spring conservative properties and the dashpot dissipative properties. The wave equation and the dispersion relation including the internal friction only are deduced using the mathematical apparatus of the fractional derivatives. Then, a new two-phase equation of state is deduced, where a part of the fluid is considered to be associated with a solid phase and another part is mobile. Substitution of the equation of state into the wave equation results in a quadratic dispersion relation whose roots yield the wave numbers of two types of the waves, namely the fast and slow ones (Grain Shearing + Effective Compressibility or GS + EC theory). According to the GS + EC theory, significant sound speed dispersion and deviations of the attenuation frequency dependence upon the linear law in the mid-frequency range occur due to a change of the medium compressibility.

Results. The results of the GS + EC theory are compared with the experimental measurement data on of phase velocity and attenuation taken from the open sources. It is shown that at low frequencies, the unconsolidated medium is of greater compressibility than at high ones that is a result of the fluid displacement from the pore space narrowing.

Discussion and conclusion. Viscous dissipation is manifested at the medium frequencies, when the fluid is still mobile and the viscous forces are rather significant. At high frequencies, the granules and the fluid oscillate together, and attenuation in the medium arises due to the forces of the internal friction.

Keywords: unconsolidated marine sediments, intergranular friction, viscous dissipation, phase velocity dispersion, attenuation coefficient, loss tangent.

Acknowledgements: the research is carried out at the financial support of RFBR and the city of Sevastopol within the framework of the scientific project No. 18-42-920001.

For citation: Lisyutin, V.A., 2019. Generalized Rheological Model of the Unconsolidated Marine Sediments with Internal Friction and Effective Compressibility. Physical Oceanography, [e-journal] 26(1), pp. 77-91. doi:10.22449/1573-160X-2019-1-77-91

DOI: $10.22449 / 1573-160 \mathrm{X}-2019-1-77-91$

(C) 2019, V.A. Lisyutin

(C) 2019, Physical Oceanography

Introduction. The Black Sea shallow part bottom contains not only natural and cultural resources, but also is the anthropogenic pollution collector. The main natural resource of the bottom is sand, the cultural one - archaeological artifacts. 
Monitoring of the bottom condition can provide information about the ecological state of the entire water area [1].

From the viewpoint of acoustics, the shallow water is a waveguide bounded from above by a fully reflective surface, and below - by a partially reflecting layered bottom. The main object of research in the acoustics of the shallow water is the bottom and marine sediments [2]. A porous water-saturated transition layer of the bottom tens of meters thick, consisting of unconsolidated sediments is of particular interest. Two types of elastic waves can propagate in the transition layer: longitudinal (compressional, $p$-wave) and shear ( $s$-wave) [2-4]. The acoustic properties of these waves and the bottom respectively are the phase velocities $c(f)$ (i.e., those determined at the tonal frequency in an infinite medium), the attenuation coefficients $\alpha(f)(\alpha, \mathrm{dB} / \mathrm{m}=8.69 \alpha, \mathrm{Np} / \mathrm{m})$ and their frequency dependencies.

In the shallow water acoustics, a variety of more or less efficient methods of bottom research are used [2, 3, 5-11]. All these methods in one way or another solve the problem of restoring the acoustic properties of the bottom from the sound field measured in the water layer. If measurements are carried out at the tonal frequency, the information about the medium is contained in the spatial interference structure of the field, the averaged transmission loss law or vertical mode profiles [2, 5-7]. It should be noted that tonal-spatial methods should be applied with caution to determine the acoustic properties of the sediments, since the result of the inversion depends on the initially selected layered bottom model. More informative are methods based on the pulsed source field analysis in a wide frequency band [7-10]. Here, the information about the bottom contains in the dispersion-dissipative characteristics of the waveguide modes. The adequacy of the broadband inversion results can be enhanced if the bottom profiling results revealing its layered structure are known [8].

When implementing any of the aforementioned inversion methods, it is necessary to solve the dispersion relation connecting the phase velocities of elastic waves in the bottom with the phase velocities of normal modes propagating horizontally in the waveguide [2].

Non-waveguide bottom methods of research, based on the analysis of the frequency-angular dependences of the reflection coefficient of sound, which can be determined from the board of a moving vessel using acoustic profilers [11], are also worthy of separate attention.

In the shallow water acoustics, the shear wave is considered non-propagating (liquid bottom) in the first approximation, the speed of sound in the bottom is frequency independent, and the attenuation coefficient is directly proportional to the frequency, which allows representing the wave number in the bottom as $k_{p}=\frac{\omega}{c_{p}}-i \alpha_{p}=\frac{\omega}{c_{p}}\left(1-i \beta_{p}\right)$, where $c_{p}$ is the speed of sound; $\alpha_{p}$ is the attenuation coefficient, $\mathrm{Np} / \mathrm{m} ; \beta_{p}$ is the constant loss tangent [2],

$$
\beta_{p}=\frac{\alpha_{p} c_{p}}{\omega}=-\frac{\operatorname{Im}\left(k_{p}\right)}{\operatorname{Re}\left(k_{p}\right)} .
$$

The assumption of a direct proportionality of attenuation and frequency makes it possible to extrapolate the results of laboratory measurements carried out at 
frequencies of hundreds of kilohertz to the low frequency range [3]. Such a model of sediments and bottom well corresponds to the basic model of a shallow water waveguide, where the bottom is represented as a homogeneous half-space (Pekeris Waveguide). The half-space is the averaging of all layers, so the Pekeris model is most often used nowadays [5-10].

When calculating sound fields at the tonal frequency the bottom representation in the liquid half-space form is often quite sufficient $[2,6]$. The situation changes when the task of inversion of the acoustic and physical properties of the bottom is set and calculations are required in a wide frequency band [8-11]. Here, the correct mapping of the frequency dependences of attenuation and phase velocity can be of fundamental importance.

Problem state and statement. In dry sand, earth materials and soils, the attenuation coefficient is proportional to the first power of frequency $\left(\alpha \sim \omega^{1}\right)$, or, equivalently, the loss tangent is constant $(\beta=$ const). Measurements in watersaturated media show deviations from this dependence mainly for highpermeability media, such as coarse sand $[3,4]$. Such a frequency dependence of attenuation implies at least two physical loss mechanisms. The main mechanism is internal friction, the second one - is viscous dissipation as the fluid moves relative to the granules. Internal friction leads to a directly proportional relationship between attenuation and frequency, viscous friction - to deviations from this dependence [4].

The topical problem of the marine sediment acoustics is the development of an adequate model of contact between the granules in an unconsolidated saturated medium. Known models can be divided into two categories: linear, based on Hooke's law, and micrononlinear, based on the phenomenological relationship between stress and strain.

The linear Biot-Stoll theory and its modern interpretations [12-14] are based on the linear contact model. The linear model assumes an elastic frame in an unconsolidated medium and at high frequencies shows the attenuation $\alpha \sim \omega^{1 / 2}$, which does not correspond to the measurements.

The micrononlinear contact model is the basis of the GS (Grain Shearing) and $V G S$ (Viscous $G S$ ) theory developed by M. Buckingham $[15,16]$. According to the $G S$ theory, the propagation and attenuation of elastic waves in sediments is due to friction between the granules, which gives the medium longitudinal and transverse rigidity and causes energy losses at the same time. The $G S$ theory is single-phase, neglects viscous dissipation and shows the frequency dependence of the attenuation $\alpha \sim \omega^{1}$, which corresponds to experiments only at high frequencies [15]. In the VGS theory, viscous dissipation is simulated by a rheological element similar to fluid, which does not permit to associate the input theory parameters with the physical properties of the medium [16].

Ultimately, the oceanological task of inversion is the restoration of the physical properties of the bottom, which a model is needed for that relates the acoustic and physical properties of marine sediments.

The present paper is aimed to develop a two-phase theory based on a suitable phenomenological equation of the dry media state. The theory initially takes into account only internal friction, but then involves the possibility of fluid movement 
in the concept of effective compressibility. Since the shift is not associated with a change in volume, shear waves will not be considered. A mathematically compact model of a single-phase medium can be constructed using the apparatus of fractional derivatives $[17,18]$.

The theory presented here is a development of a simpler model, described in [19], bases on similar provisions and retains all the notation adopted there.

Single-phase model of the medium. Consider a unbounded homogeneous two-component medium with a porosity $P$ consisting of mineral particles and a fluid. The equilibrium density $\rho_{m}$ and the equilibrium (suspension) bulk modulus $K_{m}$ of a two-component medium are determined by the formulas [3]: $\rho_{m}=P \rho_{f}+$ $+(1-P) \rho_{g}$,

$$
\frac{1}{K_{m(\text { ean })}}=\frac{P}{K_{f}}+\frac{1-P}{K_{g}},
$$

where $\rho_{f}, \rho_{g}, K_{f}, K_{g}-$ density and elastic moduli of the fluid and solid phase, respectively. The speed of sound in such a suspension will be equal to $c_{0}=\sqrt{\frac{K_{m}}{\rho_{m}}}$.

Suppose for now that there is no fluid movement, but there is an interaction between the granules of the medium, conventionally called microtension. The name is arbitrary because with small strains, the laws of microtension are significantly different from Coulomb dry or viscous friction, and the physical nature of microtension with small strains is currently a debatable question [15-19].

Following initially the works [17-19], the medium model as a generalized Kelvin - Voigt element (connected in parallel to a spring and a springpot - an element combining the conservative properties of the spring and dissipative properties of the dashpot) is represented (Fig. 1). The spring represents the pure (real-valued) elasticity of the two-component medium $K_{m}$, the springpot intergranular interaction, which laws assume the elastic-plastic nature of the deformation of the contacts between the granules.

Equation of the springpot state has the following form: $\sigma(t)=M \tau^{n} \frac{\partial^{n} \varepsilon}{\partial t^{n}}$, where $M$ is the $p$-wave modulus; $\tau$ is the constant mapping the balance between the energy accumulation and dissipation; $n$ - the order of the fractional derivative, determining the value of the exponent in the power law of the frequency dependence of attenuation; $0<n<1$ (stress relaxation exponent [15]). Then the mechanical stress $\sigma$ at the boundary of the elementary volume of the medium is associated with the strain by the equation of state

$$
\sigma(t)=K_{m} \varepsilon(t)+M \tau^{n} \frac{\partial^{n} \varepsilon(t)}{\partial t^{n}}
$$

where $\varepsilon=\operatorname{div}(\mathbf{u})$ is the volumetric strain of the medium; $\mathbf{u}$ is the medium displacement vector.

Consider a plane compression wave of the form $u_{x}=U_{0} \exp \left(i \omega t-i k_{p} x\right)$. Substituting $\varepsilon=\operatorname{div}(\mathbf{u})$ into equation (3), taking $x$ - component of the divergence from the resulting expression and applying the equation of motion in the form 
$\rho_{m} \frac{\partial^{2} \vec{u}}{\partial t^{2}}=\operatorname{div} \sigma$, the following fractional wave equation for the longitudinal component of the displacement vector is obtained:

$$
\rho_{m} \frac{\partial^{2} u_{x}}{\partial t^{2}}=K_{m} \frac{\partial^{2} u_{x}}{\partial x^{2}}+M \tau^{n} \frac{\partial^{n}}{\partial t^{n}} \frac{\partial^{2} u_{x}}{\partial x^{2}}
$$
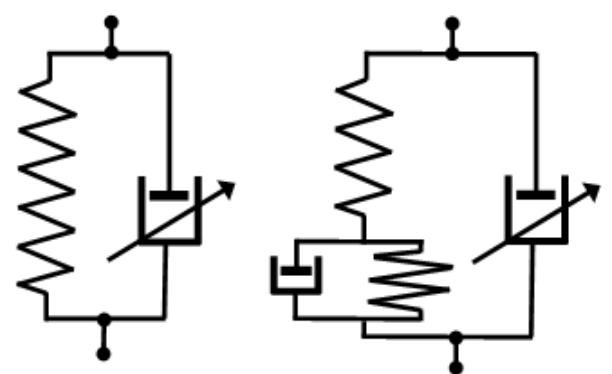

F i g. 1. Rheological models the simple non-linear contact and the one filled with fluid

Substituting the harmonic dependence into equation (4) and determining the complex phase velocity of the wave as $\tilde{c}_{p}=\frac{\omega}{k_{p}}$, the following dispersion relation is obtained

$$
\tilde{c}_{p}=\sqrt{\frac{K_{m}+\gamma\left(i \omega t_{0}\right)^{n}}{\rho_{m}}}
$$

where $\gamma=\left(\tau / t_{0}\right)^{n} M$ - equivalent rigidity of unconsolidated medium with intergranular interaction, $\mathrm{Pa} ; t_{0}=1 \mathrm{~s}$ is the constant restoring the correct physical dimension. The value of $\gamma$ can only be determined by inversion of experimental data. Equation (5) coincides with the dispersion relation of the $G S$ theory $[15,19]$.

Real sound speed $c_{p}$ and attenuation coefficients $\alpha_{p}$ can be obtained from complex phase velocities: $c_{p}=\left(\operatorname{Re}\left(\tilde{c}_{p}^{-1}\right)\right)^{-1}, \alpha_{p}=-\omega \operatorname{Im}\left(\tilde{c}_{p}^{-1}\right), \mathrm{Np} / \mathrm{m}$.

Model of quasi-two-phase medium with effective compressibility. For example, the following gedanken experiment is carried out: "compression of the medium in the jacket with drainage" [12], where the medium volume, including a many of granulas, is surrounded by an elastic jacket and is compressed by external pressure $p=-\sigma$ (3). The fluid has the ability to flow out of the jacket through the pores in the form of cylindrical tubes.

If the fluid is non-moving, does not flow out of the jacket, then the bulk modulus of the medium is determined by formula (2). If the fluid is mobile, then a part of it $\left(=\Delta V_{f}\right)$ will be squeezed out of the chosen volume $\left(=V_{u}\right)$ of the medium. This part of the fluid is represented in the form of a soft spring and a dashpot, connecting them, as shown in Fig. 1. The softness will be folded, and the compressibility of the medium have the following form [20] 


$$
K_{u}^{-1}=\frac{1}{p} \frac{\Delta V_{u}}{V_{u}}=\frac{1}{K_{m}}+\frac{1}{p} \frac{\Delta V_{f}}{V_{u}},
$$

where $V_{u}$ is the cubic volume of medium with a linear size $l$, smaller as compared with the wavelength, but larger as compared with the pore cross section. Then, instead of $K_{m}$ in equation (3) $K_{u}$ should be written: $-p=\sigma=K_{u} \varepsilon(t)+M \tau^{n} \frac{\partial^{n} \varepsilon(t)}{\partial t^{n}}$.

Consider a plane wave $p=p_{0} \exp \left(i \omega t-i k_{p} x\right)$ propagating along the $\mathrm{X}$ axis. The volume of the squeezed fluid $\Delta V_{f}$ is calculated assuming for now that the pores have a constant cylindrical section, do not intersect and are oriented along the $\mathrm{X}$ axis. Solving the Navier - Stokes equation [20], the formula for the average crosssectional pore relative velocity of fluid flow can be obtained:

$$
u_{a v}=\frac{p_{0} k_{p}\left(\rho_{g}-\rho_{f}\right)}{\omega \rho_{g} \rho_{f}}\left(1-\frac{2 J_{1}\left(i^{3 / 2} w\right)}{i^{3 / 2} w J_{0}\left(i^{3 / 2} w\right)}\right)
$$

where

$$
w=\sqrt{\frac{a^{2} \rho_{f}}{\eta} \omega .}
$$

Here $J_{0,1}$ are Bessel functions; $a$ is the pore radius; $\eta$ is the fluid dynamic viscosity, Pa.s. Denote the frequency correction function in the formula (7) as $F_{C}(w)$ :

$$
F_{C}(w)=1-\frac{2 J_{1}\left(i^{3 / 2} w\right)}{i^{3 / 2} w J_{0}\left(i^{3 / 2} w\right)} .
$$

The variation range of the real part $F_{C}(w)$ is from 0 to 1 .

The volume of fluid flowing through one of the pores is to be calculated: $\Delta V_{0}=\pi a^{2} u_{a v} T=\pi a^{2} u_{a v} \frac{l}{c_{p}}=\frac{\pi a^{2} u_{a v} l k_{p}}{\omega}$. Then, assuming that $N$ pores are contained in the chosen volume, the last term in equation (6) can be written as $\frac{1}{p} \frac{\Delta V_{f}}{V_{u}}=-\frac{k_{p}^{2}\left(\rho_{g}-\rho_{f}\right)}{\omega^{2} \rho_{g} \rho_{f}} \frac{\pi a^{2} l N}{V_{u}} F_{C}$. Substituting this expression into equation (6), the compressibility of the unconsolidated medium in the following form is obtained

$$
K_{u}^{-1}(\omega)=\frac{1}{K_{m}}-\frac{k_{p}^{2}}{\omega^{2}} \frac{\left(\rho_{g}-\rho_{f}\right)}{\rho_{g} \rho_{f}} \frac{\pi a^{2} l N}{V_{u}} F_{C}(w) .
$$

The value $P^{*}=\frac{\pi a^{2} l N}{V_{u}}$ is the geometric porosity of the medium. However, from the viewpoint of the considered dynamic fluid flow problem, the porosity $P$ included in the formula for $K_{m}$, and $P^{*}$ are different. The porosity $P$ is measured in accordance with the definition $\left(P=V_{f} / V_{\text {media }}\right)$ as the volume of fluid contained in a unit volume of medium. During laboratory measurements, sand is poured into the 
fluid, and the fluid will fill the volume of all the interstices. In a real media, part of the total pore volume is probably not included in the maze of interconnected pores, forming isolated pores in which fluid motion is absent. The fraction of the total volume of the medium formed by the pores, where the Biot flow is possible, will be called percolation porosity and denoted by $\phi$. Percolation porosity should be determined through the medium permeability [19].

Associate percolation porosity with permeability by comparing the Darcy law [3] with the Poiseuille formula, which gives

$$
\phi=\frac{s \xi \kappa}{a^{2}},
$$

where $\kappa$ is the medium permeability, $\mathrm{m}^{2} ; \xi$ is the tortuosity; $s-$ the structural coefficient, depending on the pore space geometry, for round pipes is taken $s=8$, for spherical granules $s \xi \approx 15 \div 20$.

In deriving equation (10), all cylindrical pores were assumed to be oriented along the direction of plane wave propagation. In fact, the orientation of the pores is equally likely in three directions. Inside the pores, perpendicular to the wave propagation direction, there will be no fluid motion, therefore the second term in equation (10) should be supplemented with a correction factor $S_{v}$, which value for an isotropic medium is $S_{v}=1 / 3$.

Replacing in equation (10) $P^{*}$ by $\phi$, the following expression is obtained

$$
K_{u}^{-1}=\frac{1}{K_{m}}-S_{v} \phi \frac{\left(\rho_{g}-\rho_{f}\right)}{\rho_{g} \rho_{f}} \frac{k_{p}^{2}}{\omega^{2}} F_{C}(w) .
$$

Introducing $A=\frac{\left(\rho_{g}-\rho_{f}\right)}{\rho_{g} \rho_{f}}$ in the expression (12), replacing the wave number with the complex phase velocity, the following formula is obtained

$$
K_{u}=\frac{K_{m} \tilde{c}_{p}^{2}}{\tilde{c}_{p}^{2}-S_{v} \phi K_{m} A F_{C}(w)} .
$$

Replacing $K_{m}$ in equation (5) by $K_{u}(13)$, after some algebra, a quadratic equation is obtained

$$
\rho_{m} \widetilde{c}_{p}^{4}-\left(K_{m}+\gamma D+S_{v} \phi \rho_{m} K_{m} A F_{C}\right) \tilde{c}_{p}^{2}+S_{v} \phi K_{m} \gamma D A F_{C}=0,
$$

where $D=\left(i \omega t_{0}\right)^{n}$ is the dispersion term of internal friction. The roots (14)

$$
\tilde{c}_{p}^{2}=\frac{-b \pm \sqrt{b^{2}-4 a c}}{2 a}
$$

where

$$
\begin{gathered}
a=\rho_{m}, \\
b=-\left(K_{m} \cdot\left(1+S_{\nu} \phi \rho_{m} A F_{C}\right)+\gamma D\right), \\
c=S_{\nu} \phi K_{m} \gamma D A F_{C},
\end{gathered}
$$

give squares of complex phase velocities of two types of waves - fast and slow. 
Thus, equations (14) - (18) include two sources of dispersion: internal friction, operating in the entire frequency range, and $E C$ dispersion, viscous dissipation, manifesting only in a certain frequency band. The presented theory will be called further GS +EC (Grain Shearing + Effective Compressibility).

It should be noted that equation (4) is a single phase, the constant density in the left part of (4) implies that the particles of the fluid and the solid phase oscillate together. The $G S+E C$ theory of, in contrast to [19], neglects the inertial coupling between the medium phases, revealing a new relation realized through the change in the volumetric modulus of elasticity of the medium. Inertial coupling will have effect on the shear wave dispersion, which here is considered non-propagating by agreement.

Analysis of the solution obtained. If the possibility of relative fluid motion is excluded, putting $\phi=0$, then $a=\rho_{m} ; b=-\left(K_{m}+\gamma D\right) ; c=0 ; K_{u}=K_{m}$, and formulas (15) and (5) match up, i.e. the $G S+E C$ theory goes into $G S$ one.

To subdivide the entire frequency range into low and high, in equation (8) the scaling time $\tau_{r}=a^{2} \rho_{f} / \eta$ and relaxation frequency $f_{r}==1 / \tau_{r}, \omega_{r}=2 \pi / \tau_{r}$ are defined, then the equation (8) takes the form $w=\sqrt{\omega \tau_{r}}=\sqrt{2 \pi f / f_{r}}$.

At the low $\left(f<f_{r}\right)$ frequencies $\operatorname{Re}\left(F_{C}\right) \rightarrow 0, \operatorname{Im}\left(F_{C}\right) \rightarrow 0, K_{u} \approx K_{m}$. In this case $a \approx \rho_{m}, b=-\left(K_{m}+\gamma D\right), c \approx 0$ and the $G S+E C$ theory goes into $G S$ one again, i.e. $\alpha \sim \omega^{1}$.

At seismic frequencies, dispersion can be neglected, considering $D=1$, then $c_{p 0}=\sqrt{\left(K_{m}+\gamma\right) / \rho_{m}}$ is low frequency limit of sound speed.

The minus sign in equation (15) corresponds to the slow Biot wave. In a fast wave, the medium particles oscillate in the same direction, so that the phases of compression of the solid and fluid components coincide. It is customary to say that in a slow wave, oscillations of the frame and fluid are antiphase. The physical interpretation of a slow wave in an unconsolidated medium is different. Since there is no frame, there are no long-range elastic forces that return the solid phase of the medium to the equilibrium position, therefore the slow wave is oscillating the fluid flows directed from higher to lower pressure - those diffusion extrusion flows that were taken into account in equation (6). A slow wave by stepping on watersaturated sand can be observed. Note that unlike the consolidated media in which a slow wave at high frequencies becomes propagating, in an unconsolidated one a slow wave will exist only at the lowest frequencies, and at high frequencies, on the contrary, will disappear, since the granules and the trapped fluid will oscillate together.

Comparison of the results of the $G S+E C$ theory with experimental data. The input parameters of the theory, determining the phase velocity and attenuation coefficient, are the module of intergranular rigidity $\gamma$ and the stress-relaxation exponent $n$. These parameters are determined by the experimental data inversion. The dispersion curve shape, that is, the deviation of the attenuation coefficient from the law $\alpha_{p} \sim f^{1}$, is determined by percolation porosity - the internal parameter of the model that relates the acoustic and physical properties of the medium [19]. 
Although the pores between the sand granules are clearly visible through a microscope, it is difficult to find the exact definition for a characteristic pore size. Therefore, inverting equation (11), it is assumed that $a_{0}$ is the radius of the tubular pores corresponding to the measured values of porosity $P$ and permeability $\kappa$. Then $a_{0}=\sqrt{\frac{8 \xi \kappa}{P}}$. This value will serve as the initial approximation. The best value $a$ can be determined from the inflection frequency of the dispersion curves [19].

For the case of non-intersecting pores with different sizes A. Turgut and T. Yamamoto [21] give the generalized form of the relation (11): $\frac{s \xi \kappa}{\phi a^{2}}=\chi$, $\chi=\exp \left(\sigma^{2}\right)$, where $\sigma$ is the rms deviation of pore size in case of normal law of distribution. Then $\sigma^{2}=\ln (\chi)-$ an interesting parameter for inversion, indirectly characterizing the granulometric composition of the medium.

To determine the structural coefficient $s$, we apply the Kozeny - Carman relation [3] in the form

$$
\kappa=\frac{1}{36 s \xi} \frac{P^{3}}{(1-P)^{2}} d^{2},
$$

where $d$-the average diameter of the granules. Fig. 2 shows the dependence of the measured permeability on the diameter of the granules. For this purpose, the results of permeability measurements from beads of various sizes (in Fig. 2 - circles), measurement data obtained during the experiments, which will be analyzed below (other figures), are taken from the open sources. Since the porosity of the medium from the beads depends not on the size, but only on the packing density, then, taking a random packing, it's considered $P=P_{0}=0.37$. The line constructed based on the calculations by the formula (19) gives for beads $s \xi \approx 8.25$ [19].

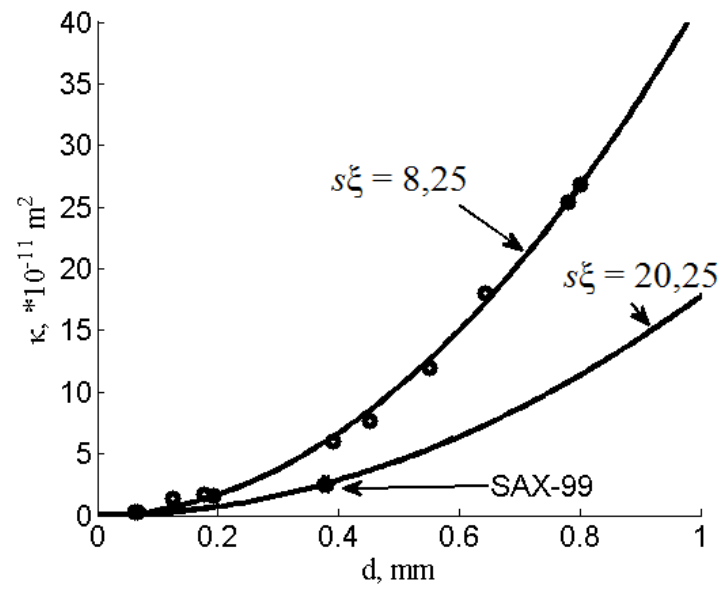

F i g. 2. Hydraulic permeability depending on the granules' diameter

First the results of the test experiment "glass beads in silicone oil" should be analyzed. Let $S_{v}=1$ here and below. The data are taken from [22]. This interesting experiment was specially carried out to test and compare the $G S$ and Biot-Stoll 
theories. Since the viscosity of silicone oil is 100 times greater than that of water, it was expected that the transition frequency, where the attenuation dependence changes from $\sim f^{2}$ to $\sim f^{1 / 2}$, will shift to higher frequencies and will be within the measurement range.

Input parameters are as follows: $d=0.4 \mathrm{~mm} ; \rho_{\mathrm{g}}=2500 \mathrm{~kg} / \mathrm{m}^{3} ; K_{\mathrm{g}}=7 \cdot 10^{10} \mathrm{~Pa}$; $\rho_{f}=968 \mathrm{~kg} / \mathrm{m}^{3} ; K_{f}=9.68 \cdot 10^{8} \mathrm{~Pa} ; \eta=98 \cdot 10^{-3} \mathrm{~Pa} \cdot \mathrm{s} ; P=0.36 ; \kappa=8.3 \cdot 10^{-11} \mathrm{~m}^{2}$; $\xi=1.7 ; a_{0}=5.25 \cdot 10^{-5} \mathrm{~m}$.

Graphs of the frequency dependences of the phase velocity, attenuation and two components, due to the internal and viscous friction effect, are shown in Fig. 3, $a$ and 3, $b$. The dispersion curves of the Biot-Stoll theory $(B-S)$ are also shown here, in Fig. 3, $a$ and 3, $b$ they coincide with the viscous component of the sound speed and attenuation.

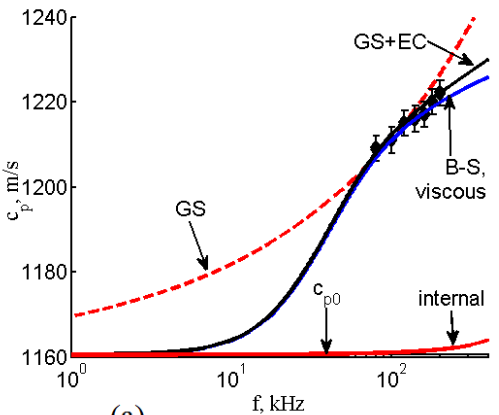

(a)

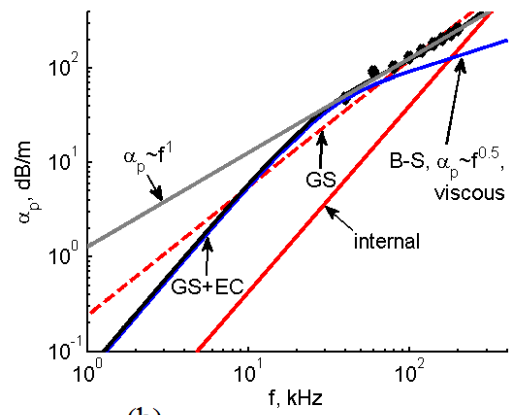

(b)

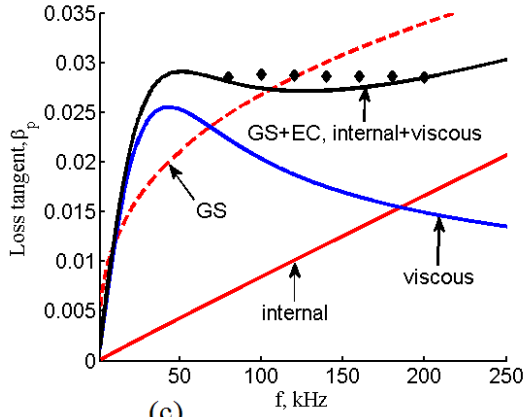

(c)

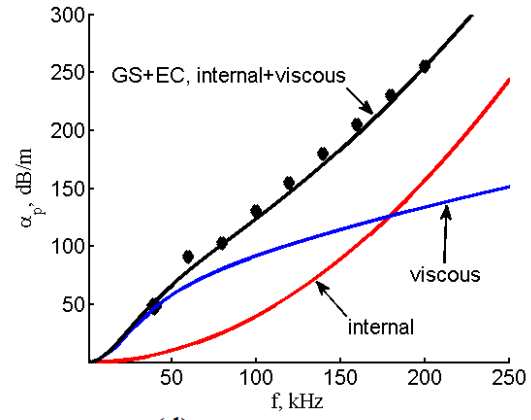

(d)

F i g. 3. Frequency dependences of sound speed $(a)$, attenuation coefficient $(b)$, loss tangent $(c)$, attenuation $(d)$ and friction components. Experiment "glass beads in the silicone oil" ( $a, b-$ the logarithmic coordinates; $c, d$ - the linear coordinates)

The inversion results are as follows: $a=0.9 a_{0} ; \gamma=98 \mathrm{~Pa} ; n=0.97 ; s \xi=8.25$; $\chi=2.7 ; \phi=0.115 ; \sigma^{2}=1.09$.

As can be seen from Fig. 3, $b$, the frequency dependence of attenuation follows the law $\alpha_{p} \sim f^{1}$. So, the authors of [22] concluded that the Biot-Stoll theory is not confirmed, and internal friction makes a decisive contribution to the total attenuation (it will be shown below that this conclusion is probably erroneous). On the other hand, the $G S$ theory is not confirmed either - the corresponding line crosses the experimental trends $c_{p}(f)$ и $\alpha_{p}(f)$, that is, it is also impossible to explain the position of the experimental points only by internal friction. The graphs of the 
$G S+E C$ theory show very good agreement, smoothly following the experimental points.

Fig. 3, $c$ and 3, $d$ show frequency dependences of two attenuation components -internal and viscous friction. The components of the velocity and attenuation were calculated, assuming in equations (17) and (18) alternately: $\phi=0$ (internal friction only); $\mathrm{n}=0$ (viscous dissipation only). Comparison of the graphs shows that only the total effect of internal and viscous friction can explain the location of the experimental points. The components of viscous and internal friction in this experiment accidentally formed in such an amazing way that the velocity dispersion is mainly due to the conservative effect of the fluid, internal friction is manifested only at the upper limit of the frequency range (Fig. 3,a). The effect of the internal and viscous attenuation components (Fig. 3, $b-d$ ) was distributed as follows: to the middle of the measurement range, the viscous dissipation effect prevails, above the middle - of the internal one, but the sum is $\alpha \sim f^{1}, \beta \approx$ const! It should be emphasized once again that the dependence $\alpha \sim f^{1}$ was obtained in this experiment, probably by accident, - such dependence shows not only internal friction, but also the sum of internal and viscous friction!

Inversion returns a low value of intergranular rigidity and a high value of the stress-relaxation exponent. Physically, this can be explained by the presence of viscous oil between the contacting surfaces, which reduces internal friction at low frequencies, but prevents this fluid from being forced out of the intergranular gap at high frequencies.

The measurement results that have the greatest practical significance for the acoustics of the shallow water are analyzed below. The results were obtained during the SAX-99 marine experiment (Sediment Acoustics Experiment) [16, 23]. The phase velocities and attenuation of the longitudinal wave were measured on site by various systems at different frequencies. Common input parameters are as follows $d=0.379 \mathrm{~mm} ; \rho_{\mathrm{g}}=2690 \mathrm{~kg} / \mathrm{m}^{3} ; K_{\mathrm{g}}=3.2 \cdot 10^{10} \Pi \mathrm{a} ; \rho_{f}=1023 \mathrm{~kg} / \mathrm{m}^{3} ; K_{f}=$ $=2.395 \cdot 10^{9} \mathrm{~Pa} ; \eta=1 \mathrm{mPa} \cdot \mathrm{s} ; P=0.385 ; \kappa=2.5 \cdot 10^{-11} \mathrm{~m}^{2} ; \xi=1.35 ; a_{0}=2,65 \cdot 10^{-5} \mathrm{~m}$.

The inversion algorithm was tuned for the best correspondence between the experimental points and the loss tangent graph (1) $\beta_{p}(f)$. Since the loss tangent depends on both sound speed and attenuation, it is more sensitive to changes in input parameters.

Graphs are shown in Fig. 4. The inversion results are as follows: $a=0.95 a_{0}$; $\gamma=1.05 \cdot 10^{8} \mathrm{~Pa} ; n=0.114 ; s=15 ; \chi=9.9 ; \phi=0.08 ; \sigma^{2}=2.29-$ the granulometric composition of the medium is quite wide, it's natural. In order for the graph to match well with the points $\beta_{p}(f)$, it is necessary to slightly deviate the theoretical line $c_{p}(f)$ upwards from the middle of the experimental trend, but the line $\alpha_{p}(f)$ goes straight along the experimental points. As can be seen from the dispersion curves, six frequency ranges can be distinguished from the relative contribution of internal and viscous friction forces [19]

1) Very low frequencies. The viscous dissipation is much less than the internal friction, attenuation $\alpha_{p} \sim f^{1}$, weak dispersion due to the $\operatorname{Re}(\gamma D)$ growth. Compressible media due to easy fluid displacement.

2) Frequency below the relaxation one. This range is in the vicinity of the first intersection point of the frequency dependence of internal and viscous friction in Fig. 4. Here, viscous dissipation is equal in magnitude to internal friction. 

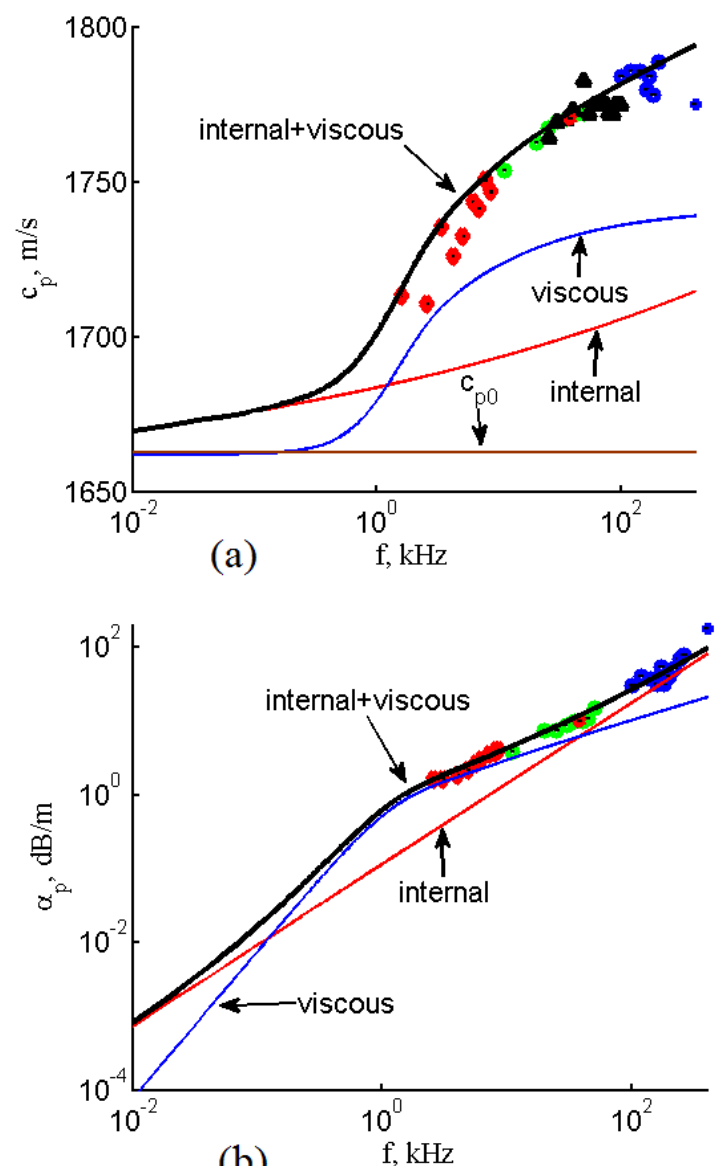

(b)

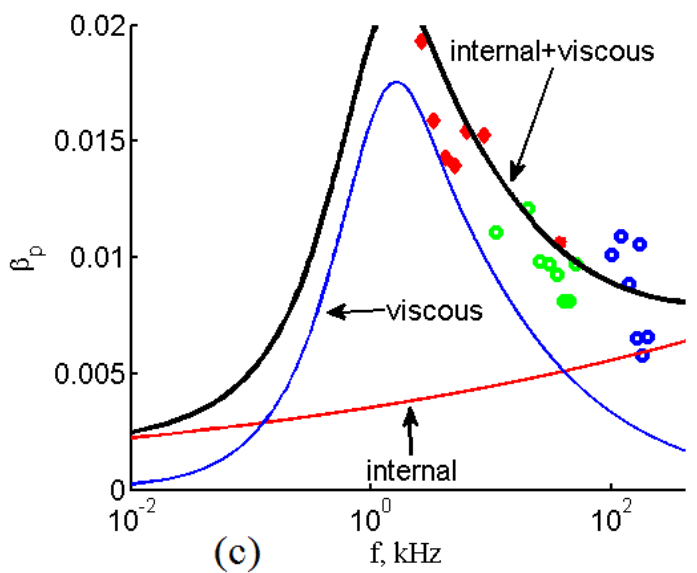

F i g. 4. Frequency dependences of sound speed $(a)$, attenuation coefficient $(b)$, loss tangent $(c)$ and friction components. $S A X-99$ experiment is a good match between the inversion results given in [10] $\left(\beta_{p}=0.0072\right.$ at frequencies about $150 \mathrm{~Hz}$ ) from Fig. 4.
The speed of sound increases, the frequency dependence of attenuation varies from $\alpha_{p} \sim f^{1}$ to $\alpha_{p} \sim f^{2}$.

3) Frequency in the vicinity of the relaxation (maximum $\beta(f)$ ). Here, the dispersion value $\partial c_{p} / \partial f$ is maximum, viscous dissipation is greater than the internal friction;

4) Frequency is higher than relaxation. This range is in the vicinity of the second intersection point of the frequency dependence of internal and viscous friction in Fig. 4. The value of the phase velocity dispersion $\quad \partial c_{p} / \partial f \quad$ sharply decreases, the frequency dependence of attenuation changes the dependence from $\alpha_{p}$ $f^{0.5}$ to $\alpha_{p} \sim f^{1}$. Viscous dissipation becomes less than the internal friction;

5) High frequencies, the upper limit of the interests of acoustics even is very shallow water. Here, weak dispersion, attenuation $\alpha_{p} \sim f^{1}$, viscous dissipation are much less than the internal friction forces;

6) Very high frequencies, over $400 \mathrm{kHz}$ - the upper limit of the $G S+E C$ theory applicability. The wavelength becomes comparable to the size of the granules, the attenuation increases due to scattering and the phase velocity decreases.

The frequency dependence of the loss tangent shown in Fig. 4, $c$, can be used for a priori estimate of losses in a similar medium at various frequencies. It should be noted that there PHYSICAL OCEANOGRAPHY VOL.26 ISS. 12019 
Conclusions and discussion. The Biot-Stoll theory has been successfully applied by oceanologists and acoustics for 50 years already, since at low and medium frequencies (up to $30 \mathrm{kHz}$ - see Fig. 4), the acoustic properties of marine sediments are correctly predicted. In fact, to take account of internal friction, R. D. Stoll adds an imaginary part to the real elasticities of the skeleton and then selects the ratio between the real and imaginary parts to obtain the correspondence with the experiment [12]. This ratio turns out to be true at the matching frequency and lower, and only at high frequencies - erroneous (see Fig. 3). During the Biot-Stoll theory scientific exploitation, a great experience has been accumulated in reconstructing the input parameters of the theory from the measured acoustic properties of the bottom [5, 11, 13, 14, 22 and 23]. The $G S+E C$ model includes all input parameters of the Biot-Stoll theory, gives the same wave field components and should become a more adequate replacement for the latter.

The $G S+E C$ theory equations are derived by replacing the single-phase equation of state (3) with a two-phase equation (6). In accordance with the latter the medium compressibility decreases with increasing frequency, which was first noticed by I.A. Chaban in [20]. The medium density was considered constant, the inertial interaction of the phases was not taken into account, and therefore, the GS + EC theory should be considered quasi-two-phase. In order for the theory to become completely two-phase, the left side of equation (4) should be changed, as in [19]. Such a transformation will give the second component of the viscous dispersion - the inertial one. It is the inertial component that determines the attenuation deviations from the law $\alpha \sim \omega^{1}$, which were also found for the shear wave [4], although to a lesser extent. If the shear wave is not considered, there is no need for complication. If the shear wave still needs to be taken into account, then the dispersion relation for it has the following form [19]: $\tilde{c}_{s}=\sqrt{\gamma_{s}\left(i \omega t_{0}\right)^{m} / \rho_{\text {eff }}}, \rho_{\text {eff }}=\rho_{m}-\phi \rho_{f} F_{C}(w) / \xi$, where $\gamma_{\mathrm{s}}$ is the shear rigidity; $m$ is shear stress relaxation exponent. The last two parameters can only be determined experimentally.

According to our ideas, the viscous dispersion mechanism in an unconsolidated medium is implemented in the following way. The fluid ability to flow from the gap between the granules increases the compressibility of the medium at low frequencies. On the other hand, as the frequency increases, the fluid viscosity appears, preventing it from being squeezed out of the gap into a more capacious part of the pore space, which leads to an increase in the medium rigidity. Thus, marine sediments exhibit not only the properties of rheopectic [18] (which will be characteristic of dry sand), but also the properties of dilatant fluids.

The $G S+E C$ model presented will be the theoretical foundation for further research of the acoustic and physical characteristics of the Black Sea shelf area bottom, united by the common scientific vector "acoustic oceanology of the shallow sea". 


\section{REFERENCES}

1. Ovsyany, E.I. and Gurov, K.I., 2016. Research of Organic Carbon and Carbonate Content in the Bottom Sediments of the Crimean Southern Coast Shelf. Physical Oceanography, [ejournal] (1), pp. 60-70. doi:10.22449/1573-160X-2016-1-60-70

2. Katsnelson, B., Petnikov, V. and Lynch, J., 2012. Fundamentals of Shallow Water Acoustics. New York: Springer, 540 p. Available at: https://b-ok.org/book/2078679/2fe5e9 [Accessed: 20 January 2018].

3. Jackson, D.R. and Richardson, M.D., 2007. High-Frequency Seafloor Acoustics. New York: Springer, 616 p. https://doi.org/10.1007/978-0-387-36945-7

4. Kibblewhite, A.C., 1989. Attenuation of Sound in Marine Sediments: A Review with Emphasis on New Low-Frequency Data. The Journal of the Acoustical Society of America, [ejournal] 86(2), pp. 716-738. https://doi.org/10.1121/1.398195

5. Zhou, J.-X., Zhang, X.-Z. and Knobles, P.P., 2009. Low-Frequency Geoacoustic Model for the Effective Properties of Sandy Seabottoms. The Journal of the Acoustical Society of America, [e-journal] 125(5), pp. 2847-2866. https://doi.org/10.1121/1.3089218

6. Grigor'ev, V.A., Lun'kov, A.A. and Petnikov, V.G., 2015. Attenuation of Sound in ShallowWater Areas with Gas-Saturated Bottoms. Acoustical Physics, [e-journal] 61(1), pp. 85-95. https://doi.org/10.1134/S1063771015010029

7. Wan, L., Zhou, J.-X. and Rogers, P.H., 2010. Low-Frequency Sound Speed and Attenuation in Sandy Seabottom from Long-Range Broadband Acoustic Measurements. The Journal of the Acoustical Society of America, [e-journal] 128(2), pp. 578-589. https://doi.org/10.1121/1.3455820

8. Belov, A.I. and Kuznetsov, G.N., 2014. Estimating the Acoustic Parameters of a Model of a Shallow-Water Seafloor Using a priori Geological and Geophysical Information and the Wigner Transform. Acoustical Physics, [e-journal] 60(2), pp. 191-196. https://doi.org/10.1134/S1063771014010047

9. Belov, A.I. and Kuznetsov, G.N., 2016. Estimating the Acoustic Characteristics of Surface Layers of the Sea Bottom Using Four-Component Vector-Scalar Receivers. Acoustical Physics, [e-journal] 62(2), pp. 194-201. https://doi.org/10.1134/S1063771016020044

10. Belov, A.I. and Kuznetsov, G.N., 2017. Spatial Attenuation of Different Sound Field Components in a Water Layer and Shallow-Water Sediments. Acoustical Physics, [e-journal] 63(6), pp. 652-659. https://doi.org/10.1134/S1063771017050037

11. Schock, S.G., 2004. A method for Estimating the Physical and Acoustic Properties of the Sea Bed Using Chirp Sonar Data. IEEE Journal of Oceanic Engineering, 29(4), pp. 1200-1217. Available http://www.eng.fau.edu/directory/faculty/schock/pdf/A_Method_for_Estimating.pdf [Accessed: 20 January 2019].

12. Stoll, R.D., 1989. Sediment Acoustics. New York: Springer, 153 p.

13. Kimura, M., 2006. Frame Bulk Modulus of Porous Granular Marine Sediments. The Journal of the Acoustical Society of America, [e-journal] 120(2), pp. 699-710. https://doi.org/10.1121/1.2211427

14. Chotiros, N.P., 2017. Acoustics of the Seabed as a Poroelastic Medium. Cham: Springer, 99 p. doi:10.1007/978-3-319-14277-7

15. Buckingham, M.J., 2000. Wave Propagation, Stress Relaxation, and Grain-to-Grain Shearing in Saturated, Unconsolidated Marine Sediments. The Journal of the Acoustical Society of America, [e-journal] 108(6), pp. 2796-2815. https://doi.org/10.1121/1.1322018

16. Buckingham, M.J., 2007. On Pore-Fluid Viscosity and the Wave Properties of Saturated Granular Materials Including Marine Sediments. The Journal of the Acoustical Society of America, [e-journal] 122(3), pp. 1486-1501. https://doi.org/10.1121/1.2759167

17. Holm, S. and Näsholm, S.P., 2011. A Causal and Fractional All-Frequency Wave Equation for Lossy Media. The Journal of the Acoustical Society of America, [e-journal] 130(4), pp. 21952202. https://doi.org/10.1121/1.3631626

18. Pandey, V. and Holm, S., 2016. Connecting the Grain-Shearing Mechanism of Wave Propagation in Marine Sediments to Fractional Order Wave Equations. The Journal of the 
Acoustical Society of America, [e-journal] 140(6), pp. 4225-4236. https://doi.org/10.1121/1.4971289

19. Lisyutin, V.A., 2018. A Simple Acoustic Model of Unconsolidated Marine Sediments with Internal Friction and Viscous Dissipation. Ecological Bulletin of Research Centers of the Black Sea Economic Cooperation, [e-journal] 15(3), pp. 39-51. https://doi.org/10.31429/vestnik-153-39-51 (in Russian).

20. Chaban, I.A., 1993. Sound Attenuation in Sediments and Rocks. Akusticheskii Zhurnal, 39(2), pp. 362-369. Available at: http://www.akzh.ru/pdf/1993_2_362-369.pdf [Accessed: 20 January 2019] (in Russian).

21. Yamamoto, T. and Turgut, A., 1988. Acoustic Wave Propagation through Porous Media with Arbitrary Pore Size Distributions. The Journal of the Acoustical Society of America, [e-journal] 83(5), pp. 1744-1751. https://doi.org/10.1121/1.396507

22. Hefner, B.T. and Williams, K.L., 2006. Sound Speed and Attenuation Measurements in Unconsolidated Glass-Bead Sediments Saturated with Viscous Pore Fluids. The Journal of the Acoustical Society of America, [e-journal] 120(5), pp. 2538-2549. https://doi.org/10.1121/1.2354030

23. Williams, K.L., Jackson, D.R., Thorsos, E.I., Tang, D. and Schock, S.G., 2002. Comparison of Sound Speed and Attenuation Measured in a Sandy Sediment to Predictions Based on the Biot Theory of Porous Media. IEEE Journal of Oceanic Engineering, 27(3), pp. 413-428. doi:10.1109/JOE.2002.1040928

About the author:

Victor A. Lisyutin - Associate Professor, Sevastopol State University (33, Universitetskaya St., Sevastopol, Russian Federation), Ph.D. (Phys.-Math.), vlisiutin@ mail.ru

The author has read and approved the final manuscript.

The author declares that he has no conflict of interest. 